\title{
Allergien: Ein Überblick
}

Ob Birkenpollen, Katzenhaare oder Haselnüsse: Bei Allergien reagiert der Organismus mit einer überschießenden Immunantwort auf normalerweise harmlose Stoffe. Je nachdem wo die pathophysiologischen Mechanismen ablaufen und welche Antikörperklassen und Vermittlerstoffe beteiligt sind, werden Allergien in 4 Typen unterteilt. HP Thomas Sokollik erläutert diese anhand der pathophysiologischen Abläufe und gibt Einblicke in Symptome, Diagnose und Behandlung.

Bereits vor mehr als 100 Jahren hat sich Clemens von Pirquet mit dem Phänomen der Allergie beschäftigt und diese als „eine veränderte Fähigkeit des Körpers, auf eine Fremdsubstanz zu reagieren“ [1] definiert. Diese ursprüngliche Definition war sehr allgemein gehalten und umfasste auch alle physiologischen Immunreaktionen. Heutzutage wird die Allergie in einem viel engeren Sinn definiert: „Eine Krankheit, die durch eine Immunreaktion gegenüber einem ansonsten harmlosen Antigen ausgelöst wird“" [2].

In der Europäischen Union leiden 20-30\% der Bürger an einem allergischen Schnupfen, 5-15\% an allergischem Asthma und 5-10\% an einem allergischen Handekzem; hinzu kommen noch 3-5\%, die an Neurodermitis leiden, wahrscheinlich als Ausdruck allergischer Reaktionen.

\section{Was ist eine Allergie?}

Die Allergie gehört zu den Immunreaktionen des Körpers, oder genauer gesagt des Immunsystems, die als Hypersensibilität (Überempfindlichkeitsreaktion) bezeichnet wird. Dabei reagiert der Organismus mit einer überschießenden Immunantwort auf körperfremde, oft harmlose Substanzen. Diese Antigene, die eine Allergie auslösen, werden auch als Allergene

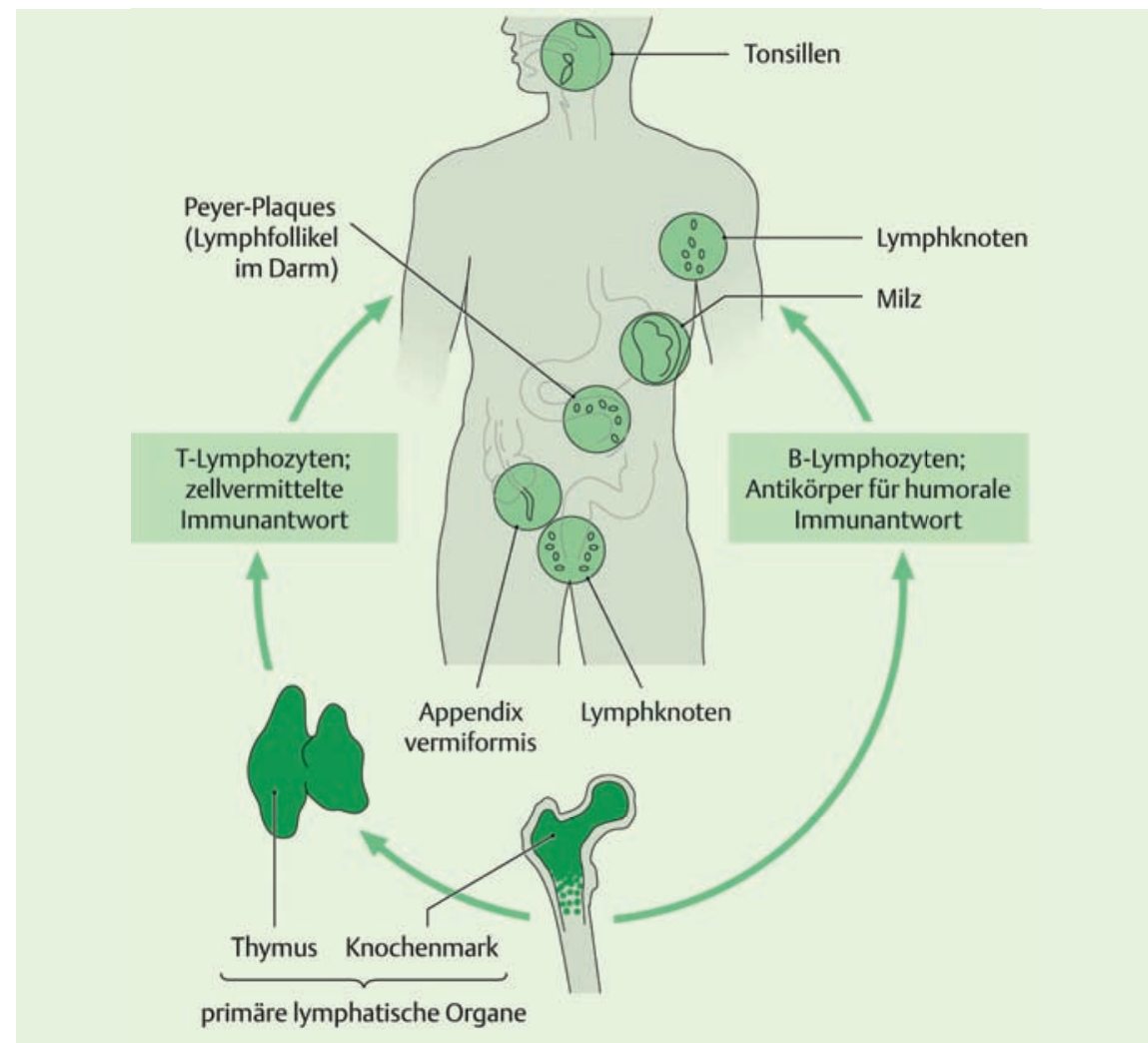

Abb. 1 Aus den lymphatischen Stammzellen bilden sich die B-Lymphozyten im Knochenmark und die T-Lymphozyten im Thymus heraus. Beide sind für die Immunreaktion verantwortlich. Quelle: $\odot$ aus [10]

bezeichnet. Bei diesen Substanzen kann es sich entweder um Mikroorganismen (Bakterien, Viren, Pilze oder Parasiten) oder eben auch als fremd identifizierte Makromoleküle handeln.

\section{Wie funktioniert die Immun- abwehr?}

Um die allergischen Reaktionsmechanismen des menschlichen Körpers zu verstehen, soll an dieser Stelle das Immunsystem näher beleuchtet werden. Das Immunsystem des Menschen besteht aus Zellen, die die Antigene in bestimmten Prozessen „bewerten“ und unschädlich machen [3]. Bei der Immunabwehr spielen die zu den
Leukozyten gehörenden Lymphozyten eine entscheidende Rolle. Aus den lymphatischen Stammzellen bilden sich die B-Lymphozyten im Knochenmark und die T-Lymphozyten im Thymus heraus. Beide sind für die Immunreaktion verantwortlich.

\section{B-Lymphozyten: spezifische humo- rale Immunabwehr}

Beim Erstkontakt (Sensibilisierung) mit einem Antigen wandelt sich ein Teil der BLymphozyten in Plasmazellen um. Diese bilden dann spezifische Antikörper aus und setzen sie frei. Plasmazellen befinden sich im Bindegewebe und haben eine Lebensdauer von 2-3 Tagen. Der andere Teil der 
B-Lymphozyten wandelt sich bei jenem Erstkontakt in teilungsfähige B-Gedächtniszellen um. Diese sind langlebig, $z$. T. über mehrere Jahrzehnte. Die Antikörper der BGedächtniszellen sind membrangebunden.

Bei einem Zweitkontakt mit dem Antigen kommt es zu einer starken Vermehrung der B-Gedächtniszellen, zu Umwandlungsprozessen in Plasmazellen und zur raschen Produktion großer Mengen Antikörper.

\section{T-Lymphozyten: spezifische zellu- läre Immunabwehr}

Beim Erstkontakt mit einem Antigen wandelt sich ein Teil der T-Lymphozyten in TEffektorzellen um. Diese T-Effektorzellen können andere, von dem Antigen befallene Körperzellen zerstören, sie sind zytotoxisch. Der andere Teil der T-Lymphozyten wandelt sich beim Erstkontakt in langlebige T-Gedächtniszellen um, die im Blut zirkulieren und darauf spezialisiert sind, das Antigen wiederzuerkennen.

Beim Zweitkontakt mit dem gleichen Antigen vermehren sich die T-Gedächtniszellen sehr stark und bilden rasch eine große Zahl an T-Effektorzellen (Sekundärantwort). Die T-Effektorzellen sind darauf spezialisiert, körpereigene Fresszellen (die das Antigen in sich aufgenommen haben) anzugreifen.

Bei den T-Effektorzellen werden unterschieden:

n- T-Killerzellen: die eigentlichen Träger des Zytotoxins

Tab. 1 Klassifikation von Allergien.

\begin{tabular}{|l|l|}
\hline Typ & $\begin{array}{l}\text { Soforttyp (Antikörper- } \\
\text { vermittelte Allergie) }\end{array}$ \\
\hline Reaktionstyp & I (anaphylaktisch) \\
\hline Antikörper & $\begin{array}{l}\text { IgE } \\
- \text { auf Mastzellen fixiert } \\
\text { - gegen spezielles Antigen }\end{array}$ \\
\hline Mediator & $\begin{array}{l}\text { Histamin, Serotonin, Brady- } \\
\text { kinin aus Mastzellen }\end{array}$ \\
\hline Gewebsreak- & $\begin{array}{l}\text { vorwiegend Histamineffekt } \\
\text { - gefäßerweiternd } \\
\text { tion }\end{array}$ \\
$\begin{array}{l}\text { - ödemsteigernd } \\
\text { - kontrahierend auf glatte } \\
\text { Muskulatur }\end{array}$ \\
\hline Reaktionszeit & \begin{tabular}{l} 
Sekunden bis Minuten \\
\hline
\end{tabular}
\end{tabular}
(beim Sensibilisierten)
- T-Helferzellen: Sie kooperieren mit den B-Lymphozyten und sind u. a. für die Bildung von Plasmazellen zuständig

- T-Supressorzellen: als Regulativ der Immunantwort durch Hemmung der Antikörperproduktion

Der menschliche Körper verfügt darüber hinaus auch noch über natürliche Killerzellen. Dabei handelt es sich um große Lymphozyten (etwa 2-5 \% aller Lymphozyten im Blut), die ebenfalls die Fähigkeit haben, Makrophagen zu zerstören.

\section{Gewebshormone und Komplement- faktoren}

Zur Steuerung der Gewebsdurchblutung, der Durchlässigkeit der Kapillargefäße, aber auch zur Regulierung der Weite der Bronchien verfügt der Körper über sog. gewebsaktive Substanzen: Histamin, Serotonin und Bradykinin. Darüber hinaus noch über Komplementfaktoren, die ähnliche Aufgaben erfüllen. All diese Substanzen werden als Immunreaktion bei Entzündungen, Gewebszerstörungen (Verbrennungen, Muskeluntergang) und auch Allergiereaktionen freigesetzt.

Die gewünschten Wirkungen sind die vermehrte Durchblutung des Gewebes (Zuführung von Serum mit entsprechenden Makrophagen und damit auch schnellerer Abtransport der Zellfragmente), aber auch eine Stimulation der Herzfrequenz, der Herzkraft und der Atmung zum selben Zweck.
Jedoch treten parallel dazu auch unerwünschte Wirkungen der gewebsaktiven Substanzen auf: Katecholaminausschüttung, Senkung des peripheren Widerstands, Senkung des onkotischen Druckgradienten und letztlich Blutdruckabfall.

Darüber hinaus löst Bradykinin Schmerzen an den Nervenendigungen aus; eine der unerwünschten Nebenwirkungen des Serotonins ist die Kontraktion glatter Muskulatur, so in Bronchien, Dünndarm und Gefäßen.

\section{Die Immunabwehr dient dem Schutz des Körpers. Ohne sie ist Leben nicht möglich. Eine überschie- ßende Reaktion des Immunsystems kann jedoch zu einer Schädigung des Organismus führen [4].}

\section{Wie werden Allergien klassifi- ziert?}

1963 klassifizierten Coombs und Gell [5] Allergien in verschiedene Typen. Heute werden Allergien üblicherweise in $\mathbf{4}$ Klassen unterteilt. Dabei wird unterschieden, welche Antikörperklassen und Vermittlerstoffe beteiligt sind und wo die pathologischen Vorgänge sich abspielen ( Tab. 1).

\section{Typ-I-Allergie}

Bei diesem Reaktionstyp, dem Anaphylaxie-Typ, lagert sich Immunglobulin $\mathbf{E}$ als Antikörper v. a. an Mastzellen und Granu-

\section{Soforttyp \\ II (zytotoxisch) \\ $\lg G, \lg M$ \\ - gegen zellständige Antigene}

aktivierte Komplementfaktoren

Zerstörung der Zellen, auf denen Antigen sitzt, durch Fresszellen, Neutrophile und Granulozyten

einige Stunden

Soforttyp
III (Immunkomplex-Reak-
tion)
IgG, IgM, IgA
- alle 3 bilden mit dem An-
tigen Immunkomplexe, die
sich an den Wandgefäßen
und Membranen ablagern
aktivierte Komplement-
faktoren
Leukozyten setzen Enzyme
frei und schädigen dadurch
umliegendes Gewebe

Minuten bis Stunden, max. 8 Stunden

\author{
Spättyp (Zell- und \\ Ekzemtyp) \\ IV (zellvermittelt) \\ spezifisch sensibilisierte \\ T-Lymphozyten \\ Lymphokine aus T-Helfer- \\ zellen \\ durch Makrophagen \\ bedingte Entzündung in \\ Leder- (Tuberkulintyp) oder \\ Oberhaut (Ekzemtyp)
}

12-72 Stunden 


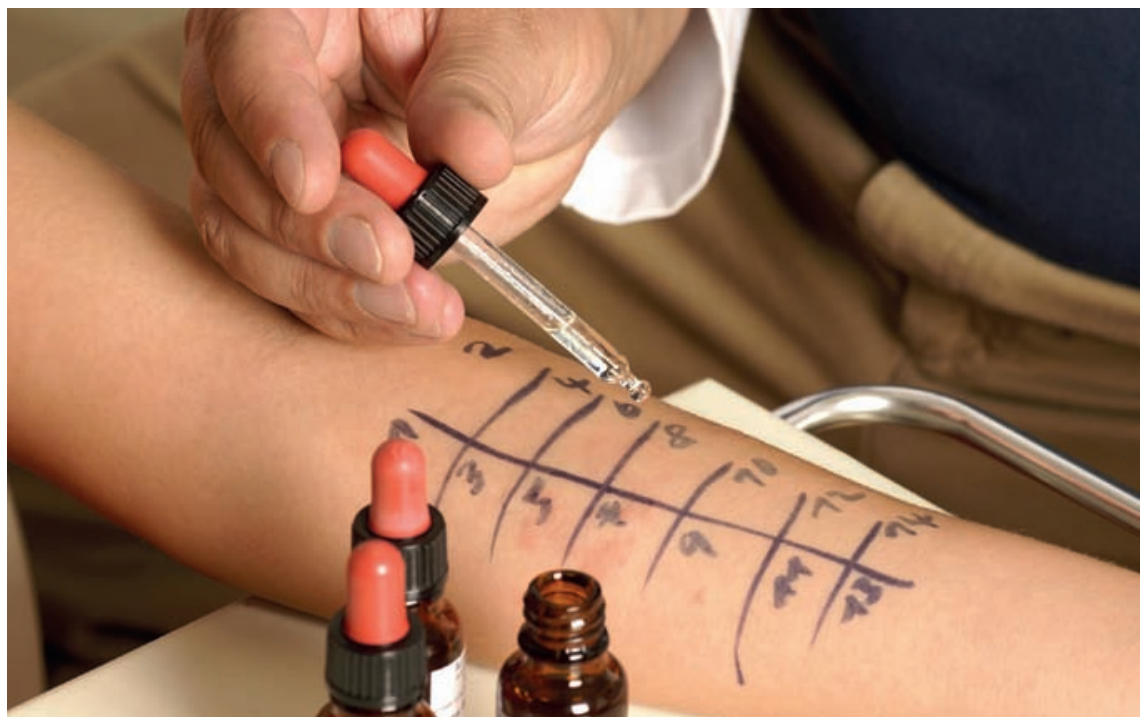

Abb. 2 Allergien werden in der Schulmedizin häufig über die Haut ausgetestet. Foto: (c Fotolia/MAST

lozyten an und führt dabei zur Freisetzung von Histamin und Serotonin. Diese beiden Mediatoren führen zu einer allergischen Sofortreaktion. Es werden auch noch Leukotriene gebildet, die über längere Zeit verengend auf die Bronchien wirken. Es kommt recht schnell zu lokalen Reaktionen. Gelangen jedoch die betreffenden Allergene in den Kreislauf, kann es zu schweren Allgemeinreaktionen bis hin zum anaphylaktischen Schock kommen. Auslösende Faktoren können z. B. sein:

- Medikamente (z. B. nicht steroidale Antirheumatika, Acetylsalicylsäure [ASS], Indometacin, Diclofenac etc.) - Pollen

- Bienen- und Wespengift

- Nahrungsmittel bzw. -zusätze

- Fremdproteine

\section{Typ-Il-Allergie}

Die Überempfindlichkeitsreaktion vom Typ II ist dadurch gekennzeichnet, dass Antikörper IgG und IgM mit Zellmembranen, v. a. auch von Blutbestandteilen, reagieren. Durch Aktivierung des Komplementsystems kommt es dann zur Zerstörung der betroffenen Zellen. Die Folge ist ein allergisch bedingter Mangel an roten bzw. weißen Blutkörperchen (Anämie, Leukopenie, Agranulozytose) oder an Blutplättchen (Thrombopenie). Allergien vom Typ II werden i. d. R. von Medikamenten ausgelöst, die an Zelloberflächen von Blutzellen wirken, z. B. Penicilline, Pyrazol, aber auch Blutderivate.

\section{Typ-III-Allergie}

Dieser Allergie-Typ führt zu einer Bildung und Ablagerung von Antigen-AntikörperKomplexen und einer Aktivierung des Komplementsystems. Dadurch entstehen örtliche Gewebsschäden. Häufige Auslöser:

- Medikamente, besonders Depotpräparate und Antibiotika, aber auch Procainamid (Neuraltherapie!)

mikrobielle Antigene

- Fremdproteine

\section{Typ-IV-Allergie}

Die allergische Reaktion vom Typ IV entspricht der zellulären Immunreaktion durch T-Lymphozyten. Zunächst werden immunkompetente Zellen selbst in den Prozess miteinbezogen. Dabei geben sie Stoffe ab, die Fresszellen anlocken, die sich dann an der Abwehrreaktion beteiligen. Beide Phasen dieses Reaktionstyps nehmen eine gewisse Zeit in Anspruch, sodass Anzeichen für eine solche Reaktion vom Typ IV frühestens 2 Tage nach Kontakt mit dem Allergen zu beobachten sind (verzögerte Immunreaktion). Die häufigsten Antigene:

1. Tuberkulintyp:

- Medikamente (z. B. Morphin, ephedrinhaltige Präparate etc.)

- mikrobielle Antigene

- Fremdgewebe (Transplantate, aber auch Fibrinkleber)

- Ekzemtyp:

- Medikamente, Salben, Berufsstoffe (z. B. Nickel, Chrom, Mangan und auch Reinigungsmittel), Kosmetika (Kontaktallergene)
Eine klare Trennung der Reaktionstypen ist nicht immer möglich, so kann es auch zu einem Übergang einer Reaktion von einem Soforttyp in einen Spättyp kommen [6].

Aktuell wird noch eine Typ-V-Reaktion differenziert. Dabei werden allergische Reaktionen durch Auto-Antikörper gegen Transmitter- oder Hormon-Rezeptoren hervorgerufen [7]. In diesem Artikel soll jedoch nicht weiter auf diesen Reaktionstyp eingegangen werden.

\section{Was sind typische Symptome?}

Typ-I- und Typ-IV-Allergien begegnen dem Heilpraktiker i. d. R. am häufigsten in der Praxis. Alleine Heuschnupfen (Typ-I-Allergie) führt in Deutschland pro Jahr zu ca. 1 Mio. Krankheitstagen.

Allergische Reaktionen sind Entzündungen. Somit stehen auch bei beiden Typen die klassischen Entzündungszeichen Rötung, Wärme, Schwellung und Schmerz (Rubor, Calor, Tumor und Dolor) im Vordergrund [8]. Gleichzeitig tritt bei den betroffenen Organen eine Functio laesa, also eine Einschränkung der Funktion ein, z. B. ist das Atmen, vielleicht sogar der Gasaustausch erschwert, bei der Haut sind Schutzfunktionen eingeschränkt bzw. außer Kraft gesetzt, sodass Sekundärinfektionen durch eindringende und sich ausbreitende Keime auftreten können.

Symptome bei Typ-I-Allergien sind (insbesondere auftretend zu bestimmten Jahreszeiten oder in bestimmter Umgebung):

- Allgemeine Symptome:

- Kopfschmerzen

- Müdigkeit

- Antriebslosigkeit

- Belastungsschwäche

Häufig vorkommende Reaktionen auf Allergene

52 \% aller Allergiker in Europa reagieren auf Gräserpollen, 49 \% auf Hausstaubmilben, 33 \% auf Pollen von Bäumen, 27 \% auf Kräuterpollen wie Ambrosia, $26 \%$ auf Tierhaare und $10 \%$ auf Schimmelpilzsporen [9]. 
- Lokale Symptome:

- Haut: Urtikaria, aber auch Angioödem

- Augen: Konjunktivitis, ggf. mit Lidödem

- Nase: Pollinosis, evtl. ödematöse Konchen

- Rachen: Kratzen im Hals, Rachenring gerötet

- Bronchien: Husten, vermehrte Schleimbildung und Auswurf

- Lunge: Asthma bronchiale

- Magen-Darm-Trakt: Meteorismus, Diarrhö

Bei Typ-IV-Allergien (Spättyp) sind als Symptome zu nennen:

nultiforme Exantheme (Tuberkulintyp)

- akute Kontaktdermatitis

- chronisches Kontaktekzem (Ekzemtyp)

\section{Wie werden Allergien} diagnostiziert?

Im Vordergrund jeder Diagnose einer Allergie-Erkrankung steht eine genaue Anamnese: Exposition am Arbeitsplatz, in der Wohnung, verwendete Stoffe (Kosmetika, Reinigungsmittel), akute und ständige Medikation, Nahrungsbestandteile, aber auch Nahrungsergänzungsmittel etc.

\section{Allergietests und Labordiagnose}

Die Austestung von Allergien geschieht sehr häufig über die Haut. Typ-I-Allergien (Sofortyp) können z. B. über einen Reibtest

\section{Praxistipp: Allergie-Tagebuch}

Häufig stellt das Auffinden des Allergens eine Sisyphusarbeit dar. Es ist ratsam, dass der Patient ein Tagebuch führt: Gewohnheiten, Speisen, alle Wege und Orte etc. sind dort aufzuführen. Zu berücksichtigen ist, dass jeder Patient, tagtäglich ungewollt mit einer Vielzahl möglicher Allergene zusammenkommt. So kann bei plötzlichem Auftreten einer Unverträglichkeit z. B. ein Konservierungsstoff in einem Lebensmittel, ein Reinigungsmittel, Waschmittel oder Shampoo gewechselt worden sein.
Labordiagnostik bei Allergien

n Differenzialblutbild: erhöhter Wert der eosinophilen Granulozyten v.a. bei allergischen Hauterkrankungen

- Gesamt-IgE: bei Atopikern erhöht, normaler Wert schließt eine Allergie (Soforttyp) jedoch nicht aus

- Bestimmung allergenspezifischer Antikörper (in Abhängigkeit der Verdachtsdiagnose):

- IgE (z. B. RAST = Radio-AllergoSorbent-Verfahren),

- IgA (z. B. Gliadin-Antikörper bei Zöliakie),

- Lymphozytentransformationstest (bei Typ IV-Allergien),

- Histamin-Release-Test (bei Typ-I-Allergien) u. a. [11]

nachgewiesen werden. Dabei wird das Allergen, z. B. Tierhaare, direkt auf die Haut des Patienten aufgetragen. Typ-IV-Allergien (Kontaktallergien) kann man mit einem sog. Patch-Test diagnostizieren. Das auszutestende Allergen (z. B. Nickel) wird in einer Trägersubstanz (häufig Vaseline) mittels eines Pflasters für ca. 48 Stunden auf der Haut des Patienten fixiert.

Zum Austesten von Typ-I-Allergien eignen sich auch sog. Perkutantests. Das Allergen wird entweder in die Haut gespritzt (Intrakutantest) oder aber durch kurzes Anstechen (Prick-Test) oder Kratzen (Scratchtest) in die Epidermis eingebracht.

Provokationstests kommen häufig in der Pulmonologie vor: Der beschwerdefreie Patient atmet das verdächtige Allergen direkt ein. Davor und danach werden die Lungenfunktionswerte spirometrisch erfasst.

\section{Provokationstests können zu schweren anaphylaktischen} Reaktionen führen und sind deshalb nur unter ärztlicher Aufsicht (oder im Krankenhaus) durchzuführen.

In bestimmten Fällen, z. B. bei vermindert belastbaren Patienten, Säuglingen und Kleinkindern etc., sind labordiagnostische Verfahren einer In-vivo-Diagnostik von Allergien vorzuziehen ( Kasten). 


\section{Verhalten bei Notfällen}

\section{Anaphylaktischer Schock}

Gelangen Allergene in den Blutkreislauf, kann es zu schweren Allgemeinreaktionen kommen (\$ S. 27). Hier muss der Heilpraktiker notfallmäßig handeln!

\section{Quincke-Ödem}

Eine Besonderheit der anaphylaktischen Reaktion ist das QuinckeÖdem. Dabei kommt es zu massiven Schwellungen des Gewebes im Gesicht, Rachen und Kehlkopf. Es droht Erstickungsgefahr. Der Heilpraktiker sollte es dringend unterlassen, Rachenraum oder sogar Kehlkopf zu inspizieren. Die Notfall-Maßnahmen entsprechen dem Vorgehen beim anaphylaktischen Schock.

\section{Naturheilkundliche Diagnose- verfahren}

Als Untersuchungsverfahren aus der $\mathrm{Na}-$ turheilkunde haben sich $z$. B. die Irisdiagnose oder der Einsatz von Bioresonanz bewährt.

\section{Irisdiagnostik}

Hilfreich kann die Irisdiagnostik sein, da sie Einblicke in den bindegewebigen Zustand des Organismus gewährt und Gewebsschwächen aufzeigt. Wie bekannt, ist eine gute Irisbefundung i.d.R. nur bei lymphatischer Konstitution und der Mischkonstitution (nach Josef Deck) möglich.

Allergische Dispositionen zeigen sich einerseits häufig in der jeweiligen Organbelastung durch entsprechende Strukturzeichen (Lakunen, Pigmente), andererseits generell in einer Belastung der BlutLymphzone (Aufhellung oder „Mottenfraß“ der Krause) bis hin zu einer Dyskrasie (als Zeichen der Belastung aller Körpersäfte).

\section{Bioresonanz- und VEGA-Testung}

Bei der Bioresonanz-Methode wird davon ausgegangen, dass die Allergene Schwingungen in den Organismus entsenden, die mit den körpereigenen Schwingungen nicht kompatibel sind. Durch entsprechende energetische Messungen soll das Allergen gefunden werden.

\section{Welche Behandlungsansätze gibt es?}

Die Behandlung von Allergien ist in der Schulmedizin auf $\mathbf{3}$ Säulen aufgebaut:

n Auslöser meiden, also den Allergenen aus dem Weg gehen

- Beschwerden lindern, i. d. R. durch den Einsatz von Antihistaminika und/ oder Kortison

- Ursachen angehen, also Hyposensibilisierung

\section{Hyposensibilisierung kritisch betrachtet}

In der Schulmedizin hat die Hyposensibilisierung einen großen Stellenwert in der Behandlung von Allergien. Über mehrere Jahre wird dem Patienten immer wieder in der allergiefreien Zeit das Allergen mit steigender Dosierung injiziert. Dadurch soll das Immunsystem das Allergen akzeptieren lernen. Der Nachteil dieser Therapie ist: Es wird verkannt, dass bei vielen Allergikern zwischenzeitlich eine Autoimmunisierung (der Organismus akzeptiert von sich aus das Allergen) und häufig ein Umschwenken auf ein anderes Allergen stattfindet.

\section{Naturheilkundliche Behandlungs- verfahren}

Die Naturheilkunde nimmt sich dem Krankheitsbild der Allergien auf vielfältige Weise an. In der Bioresonanztherapie z. B. wird das Frequenzmuster des Allergens invertiert (spiegelbildlich verkehrt) und auf den Patienten übertragen (๑ S. 39-42).

Klassisch ist hingegen das Konzept: Entgiftung - Ausleitung - Immunaufbau. Weitere Möglichkeiten der naturheilkundlichen Behandlung von Allergien sind:

1- Antihomotoxische Therapie

(D) 30-33)

- Eigenbluttherapie

- Ernährungsberatung und Ordnungstherapie

n. Homöopathie

- Kinesiologie (๑5. 19-22)

- Kolon-Hydrotherapie

nomplex-Homöopathie

- Mikrobiologische Darmsanierung

- Orthomolekulare Prinzipien

- Sauerstoff-Ozontherapie

n- Schüßler-Salze (๑ S. 56-59)
Dieser Artikel ist online zu finden unter: http://dx.doi.org//10.1055/s-0033-1337369

\section{Verwendete Literatur}

[1] Altmeyer P et al. Bildlexikon der Dermatologie. 2. Aufl. Berlin, Heidelberg, New York, Tokyo: Springer; 1995

[2] Janeway CA, Travers P. Immunologie. 2. Aufl. Heidelberg: Spektrum; 1995

[3] Ahlheim KH. Der Mensch und seine Krankheiten. 4. Aufl. Mannheim: Meyers Lexikonverlag;1988

[4] Abdolvahab-Emminger H. Exaplan. Wiesbaden: Ullstein Mosby; 1995

[5] Löffler G, Petrides P. Biochemie \& Pathobiochemie. 7. Aufl. Heidelberg: Springer, 2003

[6] Hierholzer K, Schmidt R. Pathophysiologie des Menschen, Sonderaufl. Weinheim: VCH Verlag; 1993

[7] Silbernagl S, Lang F. Taschenatlas Pathophysiologie, 3. Aufl. Stuttgart: Thieme; 2009

[8] Estler C]. Pharmakologie und Toxikologie. 3. Auflage. Stuttgart: Schattauer; 1992

[9] Bauchau V, Durham SR. Prevalence and Rate of Diagnosis of Allergic Rhinitis in Europe. European Respiratory Journal 2004; Nov; 24 (5):758-64

[10]Schünke M, Schulte E, Schumacher U. Prometheus. LernAtlas der Anatomie. Innere Organe. Illustrationen von M. Voll und K. Wesker. Stuttgart: Thieme; 2005.

[11]Hahn JM. Checkliste. Innere Medizin. 5. Aufl. Stuttgart: Thieme; 2007

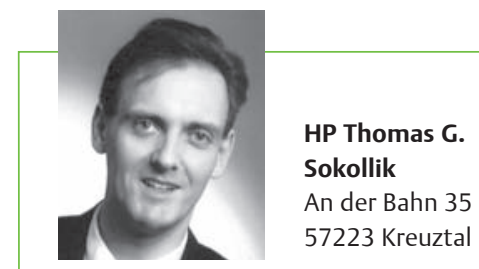

Thomas G. Sokollik hat Humanmedizin studiert. Während und nach dem Studium arbeitete er in der Anästhesie, Intensivund Rettungsmedizin. Anschließend absolvierte er ein Aufbaustudium „Social Management" und war dann über ein Jahrzehnt in der Unternehmensberatung tätig. Nun kehrte er zu seinem ursprünglichen Berufswunsch zurück und eröffnete Anfang 2013 eine Naturheilpraxis. Seine Interessen liegen in der Allergologie, Immunologie, den Erkrankungen des Bewegungsapparates und der Sportmedizin. Auch ist er als Dozent der Notfallmedizin tätig. 